\title{
Our experience of total pericardiectomy for constrictive pericarditis: a comprehensive analysis over a period of 5 years
}

\author{
Manish Jadhao, Kuntal Surana, Vijay Shewale, Chaitanya Hemant Raut, Vaibhav Shah, Prashant Mishra, \\ Jayant Khandekar
}

Department of Cardiovascular and Thoracic Surgery, Lokmanya Tilak Municipal Medical College and General Hospital, Mumbai, India

Kardiochir Torakochir Pol 2020; 17 (4): 193-197

\begin{abstract}
Introduction: Constrictive pericarditis (CP) usually presents as a result of chronic fibrous pericardial thickening and calcification of the pericardium which causes reduced cardiac output. Despite the lack of prospective studies comparing the different therapeutic strategies, surgical pericardiectomy is a valuable treatment under most circumstances.

Aim: We analyzed our records to highlight the predictors of morbidity and mortality of pericardiectomy and also short-term surgical outcome of the same procedure in a single center.

Material and methods: We carried out a comprehensive retrospective analysis of the records of patients who underwent surgery for CP at our institute between 2013 and 2018. 30 patients underwent isolated pericardiectomy. All patients underwent median sternotomy and total pericardiectomy without the use of cardiopulmonary bypass. Pre-operative, intra-operative and post-operative characteristics were noted.

Results: Fifteen patients had a history of pulmonary tuberculosis. The majority of the patients presented with NYHA grade III or IV. $60 \%$ of the patients were male. The preoperative mean central venous pressure was $24 \pm 9 \mathrm{~mm} \mathrm{Hg}$ and decreased to $9 \pm 5 \mathrm{~mm} \mathrm{Hg}$ after surgery. The 30-day mortality was $6.66 \%$ (2/30). Morbidity was mainly due to low-cardiac output syndrome $(n=4)$. A total of 26 patients had significant improvement in their NYHA status.

Conclusions: Although pericardiectomy for CP remains associated with some operative mortality, the short-term outcome is favorable, and surgical treatment is able to improve the functional class in the majority of survivors.
\end{abstract}

Key words: pericardiectomy, constrictive pericarditis, tuberculous pericarditis.

\section{Introduction}

Constrictive pericarditis (CP) usually presents as a result of chronic fibrous pericardial thickening and calcification of the pericardium [1]. The thickened and fibrotic pericardium causes reduced cardiac output as a consequence of impaired diastolic filling of the cardiac chambers [2]. Despite the lack of prospective studies comparing the different therapeutic strategies, surgical pericardiectomy is a valuable treatment under most circumstances [3-8]. The main benefits of this operation are the increase in survival, the relief of symptoms, and the prevention of disease progression [9-12].
Aim
The outcomes of pericardiectomy would greatly im- prove from appropriate surgical strategies and periopera- tive medical management based on the identification of perioperative risk factors [5, 13-15]. We analyzed our re-

cords to highlight the predictors of morbidity and mortality of pericardiectomy and also short-term surgical outcome of the same procedure in a single center.

\section{Material and methods}

We carried out a comprehensive retrospective analysis of the records of patients who underwent surgery for CP at our institute between 2013 and 2018. We identified 35 patients who underwent surgery for CP. Out of these 35 patients, 30 patients underwent isolated pericardiectomy and 5 patients had concomitant surgery for associated cardiac conditions along with pericardiectomy. We analyzed the records of the patients who underwent isolated pericardiectomy only.

The diagnosis of CP was made on the basis of clinical, echocardiography, radiological, surgical, and pathological criteria. Patients with tuberculosis and CP were started on anti-tuberculosis medicines as per the guidelines and were

Address for correspondence: Kuntal Surana MBBS, MS, MCh, Department of Cardiovascular and Thoracic Surgery, Lokmanya Tilak Municipal Medical College and General Hospital, Mumbai, India, e-mail: kuntalsurana@gmail.com Received: 5.06.2020, accepted: 17.11.2020. 


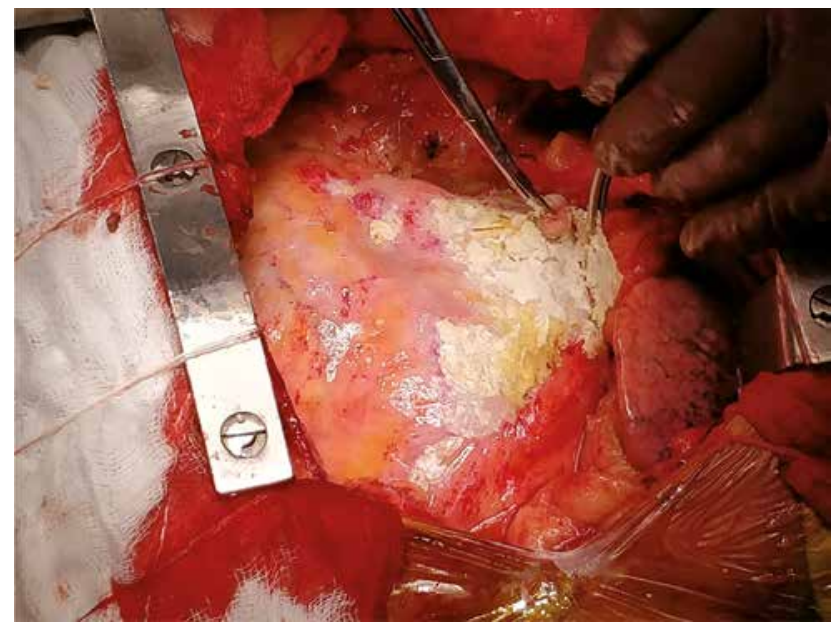

Figure 1. Intraoperative photograph of a patient with calcified pericardium

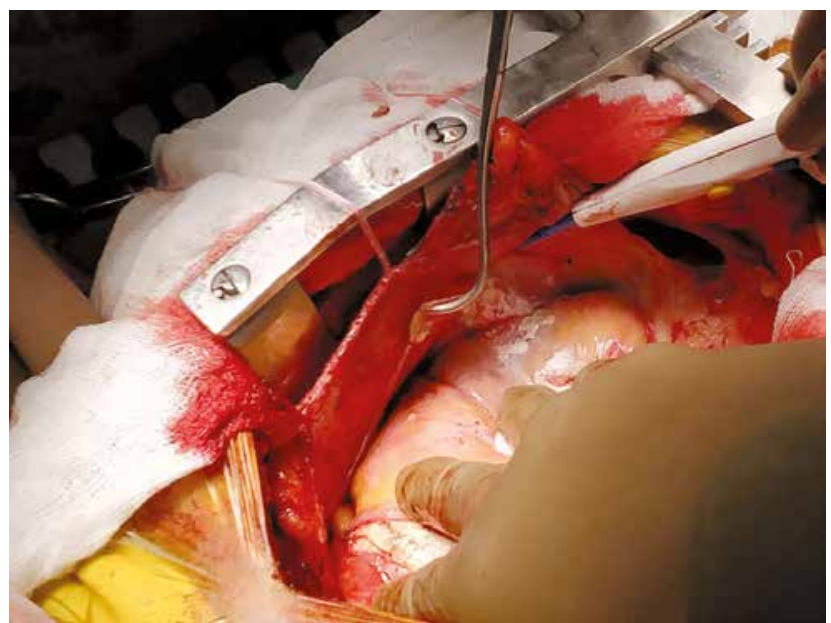

Figure 2. Intraoperative photograph of dissection being carried out

Table I. Relationship between constrictive pericarditis and the underlying causes

\begin{tabular}{lcc} 
Etiologic factors & Number & Percentage \\
Tuberculosis & 15 & 50 \\
\hline Indeterminate & 11 & 37 \\
\hline Infections other than Tuberculosis & 4 & 13 \\
\hline
\end{tabular}

Other infections included viral in 3 patients and bacterial in 1 patient.

operated on only after the intensive phase of the medicines was over. Anti-tuberculosis medicines were continued after surgery for the designated time period in the continuation phase.

All patients underwent median sternotomy. In all cases of pericardiectomy, a circuit for extracorporeal circulation was ready to use, and the perfusionist was present in the operating room. External defibrillation pads were always installed before the operation. All patients underwent total pericardiectomy, which was defined as phrenic-to-phrenic excision of the pericardium and from the great arteries superiorly to the diaphragmatic surface inferiorly [16]. The pericardium was palpated to identify a relatively soft and uncalcified area after median sternotomy, and the thymus was removed laterally. An incision was made over the pericardium. Dissection was started at the base of the aorta, extended downwards to the lateral and posterior walls of the left ventricle and the pulmonary veins, followed by the diaphragmatic pericardium. The pericardium over the right atrium and venae cavae was resected last. The myocardium was then exposed, to achieve mobilization of the heart down to the phrenic nerves. Dissection and pericardiectomy beyond the phrenic nerves was not needed in any of the patients. In the presence of dense adhesions impossible to separate we incised the area in a grid pattern carefully not to cause any injury. If calcified plaques penetrating the epicardium were present, we had to leave small islands of calcified pericardium. Cardiopulmonary bypass was not needed in patients who underwent isolated pericardiectomy (Figures 1 and 2).

Pre-operatively causes of CP were assessed, baseline clinical details of the patients were noted, and investigations were noted. Intra-operative findings, central venous pressure before and after surgery and causes of perioperative morbidity and mortality were noted. One year survival and improvement in functional class were assessed.

\section{Statistical analysis}

Results are the mean \pm standard deviation or percentages. For risk analyses, a multiple logistic regression model was developed using a forward stepwise variable selection method. The Fisher exact test or chi square test was used for categorical variables. Student's t-test was employed for continuous covariates. P-values $\leq 0.05$ were considered significant. Analyses were undertaken using SPSS version 23 by IBM.

\section{Results}

Table I shows the causes of CP and their distribution in our study. Fifteen patients had a history of pulmonary tuberculosis. The cause was not determined in 11 patients and in 4 patients some other infection was the cause. Table II summarizes the baseline clinical details of the patients. The majority of the patients presented with NYHA grade III or IV. $60 \%$ of the patients were male.

Echocardiography revealed a thickened pericardium in all patients. Preoperative computed tomography (CT) was done in all patients, and part of the pericardium was demonstrated to be thickened or calcified.

Surgical findings included a markedly thickened pericardium with some calcification in $20 / 30$ patients, caseous necrosis in the pericardial cavity in $18 / 30$ patients and a calcified pericardium penetrating the myocardium in $6 / 30 \mathrm{pa}$ tients. The preoperative mean central venous pressure was $24 \pm 9 \mathrm{~mm} \mathrm{Hg}$ and decreased to $9 \pm 5 \mathrm{~mm} \mathrm{Hg}$ after surgery ( $p<0.001)$. Surgical pathology specimens were examined for granulomas (10/30), as well as inflammation and fibrin deposition (17/30). All bacterial cultures were negative.

There were 2 deaths and both were cardiac-related. They occurred in the perioperative period as a result of 
low cardiac output syndrome due to right-heart failure. The 30-day mortality was $6.66 \%$ (2/30). Morbidity was due to low-cardiac output syndrome $(n=4)$, acute renal failure $(n=1)$, respiratory insufficiency $(n=2)$, mediastinitis $(n=1)$ and re-exploration for bleeding $(n=1)$ (Table III). A total of 26 patients had significant improvement in their NYHA status with almost all discharged patients remaining in NYHA class I/II during follow up. One-year survival in 28 patients was $92.86 \%$.

The multivariate analysis of composite surgical mortality or major morbidity is summarized in Table IV. Multivariate analysis showed that preoperative NYHA class (odds ratio $(\mathrm{OR})=2.54 ; p<0.001)$, age $(\mathrm{OR}=3.78 ; p=0.01)$, tuberculosis $(O R=20.25 ; p<0.001)$ and renal failure $(O R=2.73$; $p=0.02)$ after surgery were each positively associated with increased mortality or major morbidity.

\section{Discussion}

The clinical outcome of pericardiectomy for CP remains constrained by high peri-operative mortality. Our study has highlighted that some pre-and peri-operative factors, mainly related to the clinical condition of the patients, can adversely affect the short-term outcome. Idiopathic or viral pericarditis is the predominant cause in the western world, but tuberculosis is still a common cause of CP in "developing" and "underdeveloped" countries (especially in Asia) $[2,5,14,17]$. Patients with traumatic $C P$, post-surgery $C P$ and radiation-induced heart disease were not found in our study. On the other hand, $50 \%$ of our patients had tuberculosis. Mean age in our study was 32 years, which is about 10 years younger than that reported in earlier studies [1315]. This finding reflects the difference in the etiology of CP.

In our study CP was more common in males, which is consistent with other series [5, 13-15, 18]. Also there were almost twice as many males as females. No single approach should be used to diagnose all cases of CP [19]. The diagnostic approach should be individualized for patients. The prominent signs are pleural effusions, ascites, leg edema, and increased jugular venous pressure. Sometimes, however, patients may not present with sufficient signs or symptoms for a definitive diagnosis to be made. In such cases, additional imaging is required and recommended.

Echocardiography should be the initial noninvasive imaging employed [5, 17, 19-21]. CT and MRI give more information than echocardiography and should be utilized wherever available. We followed the same approach and CT was done in all the patients. CT helped us to identify pericardial calcification more accurately and we were able to plan our intra-operative strategy with more ease.

Pericardiectomy is the most effective curative treatment for chronic CP $[5,13-15,17]$. In the current study preoperative NYHA grade was positively associated with mortality and major morbidity, and hence as per our experience patients with CP should be operated on as soon as the diagnosis is made and before they develop NYHA grade III heart failure. In our institute, pericardiectomy is carried out within 1 week after the diagnosis, before clinical manifesta-
Table II. Pre-operative baseline clinical details

\begin{tabular}{lc} 
Variable & Results \\
Age [years] & $32 \pm 15.5$ \\
\hline Males & $18(60 \%)$ \\
\hline Diabetes & $2(6.6 \%)$ \\
\hline Renal dysfunction & $1(3.3 \%)$ \\
\hline NYHA class: & $4(13.3 \%)$ \\
\hline II & $18(60 \%)$ \\
\hline III & $8(26.6 \%)$ \\
\hline Raised jugular venous pressure & $21(70 \%)$ \\
\hline Lower limb edema & $20(66.66 \%)$ \\
\hline Abdominal distension & $20(66.66 \%)$ \\
\hline Palpitation & $12(40 \%)$ \\
\hline Smoking & $10(33.33 \%)$ \\
\hline Hypoalbuminemia & $12(40 \%)$ \\
\hline Pleural effusion & $22(73.33 \%)$ \\
\hline Pulmonary infiltrates & $15(50 \%)$ \\
\hline Pericardial calcification & $6(20 \%)$ \\
\hline Rhythm disturbances & $14(46.66 \%)$ \\
\hline
\end{tabular}

Age is given as range and other characteristics are given as percentage.

Table III. Intra-operative and postoperative findings for the surgery

\begin{tabular}{lc} 
Variable & Results \\
\hline CVP change [mm Hg]: & $24 \pm 9$ \\
\hline Pre-operative CVP & $9 \pm 5$ \\
\hline Postoperative CVP & $1(3.33)$ \\
\hline Re-exploration for bleeding & $4(13.33)$ \\
\hline Low output syndrome & $1(3.33)$ \\
\hline Renal failure & $2(6.66)$ \\
\hline Mediastinitis & $1(3.33)$ \\
\hline Intensive care unit stay [days] & $3 \pm 2$ \\
\hline Hospital stay [days] & $21 \pm 10.3$ \\
\hline In-hospital mortality & $2(6.66)$ \\
\hline
\end{tabular}

CVP - central venous pressure, CVP - intensive care unit and hospital stays are in range and the other parameters are in percentage in brackets.

Table IV. Multivariate predictors of composite surgical mortality and major morbidity

\begin{tabular}{lcc} 
Covariate & Odds ratio & P-value \\
Age & 3.78 & 0.01 \\
\hline Male sex & 0.55 & 0.99 \\
\hline Diabetes & 0.90 & 0.58 \\
\hline Tuberculosis & 20.25 & $<0.001$ \\
\hline NYHA class & 2.54 & $<0.001$ \\
\hline Renal failure & 2.73 & 0.02 \\
\hline
\end{tabular}

tions become worse. Various approaches and methods (left anterolateral thoracotomy; median sternotomy; $U$ incision with the base of the $U$ lying at the left sternal border; bilateral thoracotomy) have been described since Rehn and 
Sauerbruch conducted a successful pericardial resection for chronic CP [22]. The most commonly used approach is median sternotomy, which is also the approach of choice at our institute. Median sternotomy provides more radical clearance of the pericardium over the right atrium and venae cavae, and allows extensive pericardial removal and also facilitates use of cardiopulmonary bypass if needed. $[13,20]$.

Chowdhury et al. suggested that delayed improvement and persistent symptoms are most commonly the result of incomplete decortications [5]. However, Schwefer et al. suggested that the long-term outcome is related not only to extent of the surgery but also the etiology of pericardial disease and preoperative NYHA status [19]. In the current study, despite total pericardiectomy, $6.66 \%$ of patients had early postoperative deaths due to low-output syndrome.

A correlation between NYHA grade and overall or early survival has been observed by our study and also other studies, and hence early pericardiectomy is advocated [23]. In the current study, low-output syndrome and respiratory insufficiency were the most common postoperative complications. Improved perioperative management and medical therapy are important to avoid low cardiac output and restoration of right-heart function. Diuretics, inotropes, and vasodilators are the best medical therapies in the perioperative period [17]. However, there is a temptation, if faced with a much raised venous pressure and edema, for overzealous use of diuretics. Such management can lead to sudden death due to electromechanical dissociation. Also care should be taken to start proper treatment for the etiology pre-operatively.

In the current study tuberculosis was the most common etiology for chronic CP and we started our patients on anti-tuberculosis medications pre-operatively as per guidelines. These medicines were continued postoperatively for a period of 6-9 months. Tuberculous CP can have significant involvement of the lungs [2]. Preoperative chronic lung disease also has a considerable negative effect on postoperative results. Effective interventions can include aggressive physiotherapy, fiberoptic bronchoscopy along with aggressive screening for postoperative pneumonia. Blood transfusion may be needed for CP patients suffering from tuberculosis due to long-term malnutrition and deprivation of cardiogenic nutrients [14]. Also tuberculosis was positively associated with mortality in the present study. Hence proper management of tuberculosis is a must for a good surgical outcome.

The current study is a retrospective observational study with a small cohort and analysis of short-term results. Long-term follow-up data on survival are lacking, which limits our ability to understand the long-term benefits of total pericardiectomy. We found mostly infective etiology for chronic CP in the current study and the outcome of pericardiectomy for this etiology was analyzed. We do not know the outcome of pericardiectomy for other etiological factors.

\section{Conclusions}

Although pericardiectomy for CP remains associated with some operative mortality, the short-term outcome is favorable, and surgical treatment is able to improve the functional class in the majority of survivors. Some preoperative and peri-operative factors, mainly related to the patients' clinical conditions, can adversely affect the short-term outcome. Careful perioperative management and surgical intervention upon or before right-heart failure may improve outcome. This is accomplished more readily through median sternotomy in patients with CP. Routine use of cardiopulmonary bypass during pericardiectomy is not necessary.

\section{Disclosure}

The authors report no conflict of interest.

\section{References}

1. Brockington GM, Zebede J, Pandian NG. Constrictive pericarditis. Cardiol Clin 1990; 8: 645-661.

2. Tokuta Y, Miyata H, Motomura N, Araki Y, Oshima H, Usui A, Takamoto S; Japan Adult Cardiovascular Database OrganizationOutcome of pericardiectomy for constrictive pericarditis in Japan: a nationwide outcome study. Ann Thorac Surg 2013; 96: 571-576.

3. Szabo G, Schmack B, Bulut C, Soós P, Weymann A, Stadtfeld S, Karck M. Constrictive pericarditis: risks, etiologies and outcomes after total pericardiectomy: 24 years of experience. Eur J Cardiothorac Surg 2013; 44: 1023-1028.

4. Avgerinos D, Rabitnokov Y, Worku B, Neragi-Miandoab S, Girardi LN. Fifteenyear experience and outcomes of pericardiectomy for constrictive pericarditis. J Card Surg 2014; 29: 434-438.

5. Chowdhury UK, Subramaniam GK, Kumar AS, Airan B, Singh R, Taiwar S, Seth S, K Mishra P, Pradeep KK, Sathia S, Venugopal P. Pericardiectomy for constrictive pericarditis: a clinical, echocardiographic, and hemodynamic evaluation of two surgical techniques. Ann Thorac Surg 2006; 81: 522-529.

6. George TJ, Arnaoutakis GJ, Beaty CA, Kilic A, Baumgartner WA, Conte JV. Contemporary etiologies, risk factors, and outcomes after pericardiectomy. Ann Thorac Surg 2012; 94: 445-451.

7. Bertog SC, Thambidorai SK, Parakh K, Schoenhagen P, Ozduran V, Houghtaling PL, Lytle BW, Blackstone EH, Lauer MS, Klein AL. Constrictive pericarditis: etiology and cause-specific survival after pericardiectomy. J Am Coll Cardiol 2004; 43: 1445-152.

8. Ni Y, von Segesser LK, Turina M. Futility of pericardiectomy for post irradiation constrictive pericarditis? Ann Thorac Surg 1990; 49: 445-448.

9. Somerville W. Constrictive pericarditis. With special reference to the change in natural history brought about by surgical intervention. Circulation 1968; 38: 102-111.

10. Seifert FC, Miller DC, Oesterle SN, Oyer PE, Stinson EB, Shumway NE. Surgical treatment of constrictive pericarditis: analysis of outcome and diagnostic error. Circulation 1985; 72: II264-II273.

11. Nataf P, Cacoub P, Dorent R, Jault F, Bors V, Pavie A, Cabrol C, Gandjbakhch I. Results of subtotal pericardiectomy for constrictive pericarditis. Eur J Cardiothorac Surg 1993; 7: 252-255.

12. Astudillo R, Ivert T. Late results after pericardiectomy for constrictive pericarditis via left thoracotomy. Scand J Thorac Cardiovasc Surg 1989; 23: 115-119.

13. Gopaldas RR, Dao TK, Caron NR, Markley JG. Predictors of in-hospital complications after pericardiectomy: a nationwide outcomes study. J Thorac Cardiovasc Surg 2013; 145: 1227-1233.

14. Lin YY, Zhou M, Xiao J, Wang B, Wang ZN. Treating constrictive pericarditis in a Chinese single-center study: a five-year experience. Ann Thoarc Surg 2012; 94: $1235-1240$.

15. Ling LH, Oh JK, Schaff HV, Danielson GK, Mahoney DW, Seward JB, Tajik AJ. Constrictive pericarditis in the modern era evolving clinical spectrum and impact on outcome after pericardiectomy. Circulation 1999; 100: 1380-1386. 
16. Chowdhury UK, Narang R, Malhotra P, Choudhury M, Choudhury A, Singh SP. Indications, timing and techniques of radical pericardiectomy via modified left anterolateral thoracotomy (UKC's modification) and total pericardiectomy via median sternotomy (Holman and Willett) without cardiopulmonary bypass. J Pract Cardiovasc Sci 2016; 2: 17-27.

17. Ghavidel AA, Gholampour M, Kyavar M, Mirmeshdag Y. Constrictive pericarditis treated by surgery. Tex Heart Inst J 2012; 39: 199-205.

18. Sengupta PP, Eleid MF, Khandheria BK. Constrictive pericarditis. Circ J 2008 72: 1555-1562.

19. Schwefer M, Aschenbach R, Heidemann J, Mey C, Lapp H. Constrictive pericarditis, still a diagnostic challenge: comprehensive review of clinical management. Eur J Cardiothorac Surg 2009; 36: 502-510.
20. Talreja DR, Edwards WD, Danielson GK, Schaff HV, Tajik AJ, Tazelaar HD, Breen JF, Oh JK. Constrictive pericarditis in 26 patients with histologically normal pericardial thickness. Circulation 2003; 108: 1852-1857.

21. Skubas NJ, Beardslee M, Barzilai B, Pasque M, Kattapuram M, Lappas DG. Constrictive pericarditis: intraoperative hemodynamic and echocardiographic evaluation of cardiac filling dynamics. Anesth Analg 2001; 92: 1424-1426.

22. Glenn F, Diethelm A. Surgical treatment of constrictive pericarditis. Ann Surg 1962; 155: 883-893.

23. Tirilomis T, Unverdorben S, von der Emde J. Pericardiectomy for chronic constrictive pericarditis: risks and outcome. Eur J Cardiothorac Surg 1994; 8: 487-492. 